\title{
Comments on "Endoscopic Sign in Sigmoid Volvulus with Mucosal Ischemia: Autumn Leaves"
}

\author{
Sabri Selcuk Atamanalp ${ }^{1}$ (D) \\ Received: 4 June 2020 / Accepted: 30 July 2020 /Published online: 5 August 2020 \\ (C) Association of Surgeons of India 2020
}

Dear editor,

I read with great interest the article by Uylas et al. [1], who reported a patient with sigmoid volvulus (SV) with borderline mucosal ischemia treated non-operatively. Although SV, the wrapping of the sigmoid colon around its own base causing an intestinal obstruction, is rare worldwide, it is endemic in Turkey, my practicing area. My colleagues and I have a 1028-case experience with SV over a 53.5-year period from June 1966 to January 2020, which is the largest single-center SV series over the world [2]. Here, I would like to discuss the new description of the authors for borderline ischemic mucosa, autumn leaves, and the treatment options in borderline sigmoid ischemia in SV.

First, sigmoid gangrene, which develops in $6.1-93.4 \%$ of cases, is a catastrophic complication of SV. Endoscopic signs of bowel gangrene include devitalized black-deep brown mucosa with gangrenous-bloodstained effluent, which can easily be distinguished from light pink viable mucosa [3]. Conversely, in my experience, the evaluation of the borderline ischemic mucosa is more difficult. For this reason, the authors' new description for the borderline ischemic mucosa, "fallen leaves in autumn" is a perfect definition for black mucosa including yellow-reddish spots.

Second, in SV, the therapeutic strategy is well established in patients with viable bowel, in whom endoscopic decompression followed by elective sigmoid colectomy is advised. Similarly, the treatment of the patients with gangrenous bowel is also well discussed, in whom emergency surgical resection is required [2]. However, there is no available data in literature on the treatment of borderline sigmoid ischemia, and classical approach includes two options: emergency surgical treatment, which has high mortality and morbidity in unstable and comorbid patients, or observation with clinical and laboratorial

Sabri Selcuk Atamanalp

ssa@atauni.edu.tr

1 Department of General Surgery, Faculty of Medicine, Ataturk University, Erzurum, Turkey evaluation, which is undefinable by most investigations including Doppler ultrasonography, CT angiography, or scintigraphy. In this field, we described a novel approach in 2017, which comprises an observation with repeating endoscopies every few hours [3]. By this way, mucosal viability may come to life in some patients, while time for preoperative preparation is gained in some others. By using this method, we treated four patients with no mortality; in three of them (75.0\%), a recovery was obtained, as was in the authors' case, while one patient $(25.0 \%)$ required delayed emergency surgery. Nevertheless, the critical point in decision-making process is the patient selection for this method. In my experience, in SV patients with borderline sigmoid ischemia, cases older than 70 years old, and those with American Society of Anesthesiologists (ASA) Score $\geq$ IV, whose expected operative mortality rate is higher than $7.8 \%$, are potential candidates for observation with repeating endoscopies.

I congratulate the authors for their interesting description and practice, and I wonder about their opinion on my comments.

\section{Compliance with Ethical Standards}

Conflict of Interest The author declares that he has no conflict of interest.

\section{References}

1. Uylas U, Kutluturk K, Sumer F, Kayaalp C (2020) Endoscopic sign in sigmoid volvulus with mucosal ischemia: 'autumn leaves'. Indian J Surg. https://doi.org/10.1007/s12262-020-02341-5

2. Atamanalp SS (2019) Sigmoid volvulus: the first one thousand-case single center series in the world. Eur J Emerg Surg 45:175-176

3. Atamanalp SS, Atamanalp RS (2017) What is done when endoscopic examination reveals borderline bowel ischemia in patients with sigmoid volvulus. Pak J Med Sci 33:761-763

Publisher's Note Springer Nature remains neutral with regard to jurisdictional claims in published maps and institutional affiliations. 